\title{
BMJ Open Protocol for a randomised controlled trial for Treatment in Thoracic Aortic Aneurysm: Surgery versus Surveillance (TITAN: SvS)
}

\author{
Ming Hao Guo, ${ }^{1}$ Jehangir J Appoo, ${ }^{2}$ George A Wells, ${ }^{3,4}$ Michael Chu, ${ }^{5}$ \\ Maral Ouzounian, ${ }^{6}$ Jacqueline Fortier, ${ }^{1}$ Munir Boodhwani (1) ${ }^{1}$
}

To cite: Guo MH, Appoo JJ, Wells GA, et al. Protocol for a randomised controlled trial for Treatment in Thoracic Aortic Aneurysm: Surgery versus Surveillance (TITAN: SvS). BMJ Open 2021;11:e052070. doi:10.1136/ bmjopen-2021-052070

- Prepublication history and additional supplemental material for this paper are available online. To view these files, please visit the journal online (http://dx.doi.org/10.1136/ bmjopen-2021-052070).

Received 07 April 2021 Accepted 10 May 2021

Check for updates

(c) Author(s) (or their employer(s)) 2021. Re-use permitted under CC BY-NC. No commercial re-use. See rights and permissions. Published by BMJ.

For numbered affiliations see end of article.

Correspondence to

Dr Munir Boodhwani;

mboodhwani@ottawaheart.ca

\section{ABSTRACT}

Introduction Ascending thoracic aortic aneurysm (ATAA)

is an asymptomatic condition that can lead to catastrophic events of rupture or dissection. Current guidelines are based on limited retrospective data and recommend surgical intervention for ATAA with a diameter of greater or equal to $5.5 \mathrm{~cm}$. Treatment in Thoracic Aortic Aneurysm: Surgery versus Surveillance is the first prospective, multicentre, randomised controlled trial that compares outcomes of patients undergoing early elective ascending aortic surgery to patients undergoing medical surveillance. Methods and analysis Patients between the ages of 18 and 80 with an asymptomatic ATAA between $5.0 \mathrm{~cm}$ and $5.4 \mathrm{~cm}$ in diameter are eligible for randomisation to early surgery or surveillance. Patients in the surgery group will be followed at 1 month after discharge, then annually for a minimum of 2 years and up to 5 years. Patients in the surveillance group will be followed annually from their index clinic visit for a minimum of 2 years and up to 5 years. The primary outcome is all-cause mortality at follow-up. A sample size of 618 subjects (309 in each group) will achieve an $80 \%$ power at a 0.047 significance level.

Ethics and dissemination This study has received Ottawa Health Science Network Research Ethics Board approval (Protocol 20180007-01H), which was most recently updated on 25 November 2020. The Research Ethics Board have granted approval to the study at 14 participating institutions, including the Ottawa Health Science Network Research Ethics Board. On completion of data analysis, the result of the trial will be presented at national and international conferences, and published in relevant journals, regardless of the finding of the trial. Trial registration number NCT03536312.

\section{INTRODUCTION}

Ascending thoracic aortic aneurysm (ATAA) is known as 'silent killers', as they are usually indolent until presentation with aortic rupture or dissection. Aortic rupture is nearly always fatal, and acute type A aortic dissection has a $20 \%$ mortality within the first 24 hours and $50 \%$ at 1 week when treated medically. ${ }^{1}$ Even when patients undergo emergency
Strengths and limitations of this Study

The trial is a multicentre collaborative effort

- All imaging measurements are performed in a centralised core laboratory to minimise interobserver variation.

- The trial includes a registry arm in which the natural history of ascending aortic aneurysm could be followed.

- The recruitment and follow-up period required is long.

surgery, mortality for acute type A dissection repair remains high at $19.7 \% .^{2}$

Elective ATAA replacement can prevent the future occurrence of acute aortic events. However, elective surgery is invasive and carries risks $(1 \%-2 \%)$ of mortality and stroke. ${ }^{3}$ Therefore, the management of ATAA is predicated on recognising the threshold at which risks of surveillance outweigh risks of surgery.

The natural history of ATAA is incompletely understood. Aneurysm size is the most widely accepted criteria for predicting risk of acute aortic events and determining surgical threshold. ${ }^{45}$ Using a retrospective cohort of patients, Elefteriades and colleagues from Yale University observed a 15\%/year risk of aortic dissection, rupture or death at aortic size of $\geq 6.0 \mathrm{~cm}$ compared with $7 \% /$ year between $5.0 \mathrm{nd} 5.9 \mathrm{~cm}^{3}$. Driven primarily by this data, current guidelines recommend surgery at an ascending aortic diameter of $\geq 5.5 \mathrm{~cm}$ in the absence of connective tissue disorders, familial aortopathy or rapid growth $(>3 \mathrm{~mm} /$ year $) .{ }^{4-7}$ We have performed a metaanalysis of observational studies reporting natural history of ATAA, which included 8831 patients and $>31000$ patient-years of follow-up. ${ }^{8}$ The mean aortic diameter in these studies was $4.3 \mathrm{~cm}$ (range $3.6-5.6 \mathrm{~cm}$ ), and 
the pooled incidence of all-cause mortality was $1.99 \% /$ patient-year. In addition to providing an estimate for risk in small aneurysms, this meta-analysis confirmed that there is little prospective data and no randomised trials to inform current guidelines, which lead to over 10000 elective proximal aortic surgeries performed annually in North America. ${ }^{9}$ Furthermore, the number of patients needed to be treated with elective repair to prevent one adverse outcome is unknown.

While the risk of aortic dissection grows as aneurysm size increases, data from the International Registry of Aortic Dissection revealed that patients presenting with acute type A dissection have a median aortic diameter of $5.3 \mathrm{~cm}$, suggesting that over $50 \%$ of aortic dissections occurs in aneurysms with diameters $<5.5 \mathrm{~cm} .{ }^{10}$ Other large cohort studies have corroborated these findings that the majority of aortic dissections tend to occur at diameters less than $5.5 \mathrm{~cm}^{11}{ }^{12}$ Therefore, patients who do not meet guideline criteria for surgery still bear a significant burden of health risk and quality-of-life concern.

In contrast, treatment guidelines for abdominal aorta aneurysm are founded on extensive data and are widely accepted by the surgical community. ${ }^{13}$ Multiple large, multicentred randomised control trials that included 127891 men and 9342 women established the natural history of small abdominal aortic aneurysms $(<5.5 \mathrm{~cm})$ as well as proper surveillance for patients at risk of this life-threatening disease. ${ }^{14-18}$ In addition, by comparing long-term outcomes of patients randomised to either the surveillance group (surgery if aneurysm size $>5.5 \mathrm{~cm}$ or growth rate $>1.0 \mathrm{~cm}$ per year) or the early surgery group, the trials have shown that for Abdominal Aortic Aneurysm (AAAs) under $5.5 \mathrm{~cm}$, the risk of early surgery outweighs the risk of rupture during surveillance and that women have higher risk of rupture than men.

Treatment in Thoracic Aortic Aneurysm: Surgery versus Surveillance (TITAN: SvS) aims to be the first randomised controlled trial to guide evidence-based management of the growing population with indolent but lethal silent killers of ascending aortic aneurysms. In addition to a multicentre prospective clinical database, the trial will also include a core imaging lab and a quality of life analysis.

\section{Objectives}

The primary objective of the trial is a comparison of allcause mortality between groups undergoing surveillance versus elective ascending aortic surgery for patients with degenerative or bicuspid valve-related ascending aortic aneurysm over the study period.

The secondary objectives of the trial include operative and 30-day mortality following aortic surgery, incidence of acute aortic events, incidence of elective ascending aortic surgery in the surveillance group, aorta-related death, nonaorta-related death, incidence of cerebrovascular accidents (CVA) during follow-up, annual ascending aortic growth rate $(\mathrm{mm} /$ year) in the surveillance group, quality of life at 1 year and 3 years assessed using the SF-36 questionnaire.

\section{Trial design}

The TITAN: SvS trial is an open label, nonblinded, prospective, multicentre randomised control trial that compares all-cause mortality and aortic events in patients with ascending aortic aneurysm between 5.0 and $5.4 \mathrm{~cm}$ undergoing early elective surgery or surveillance (figure 1). Eligible patients not consenting to randomisation and those with ascending aorta $>5.5 \mathrm{~cm}$ with prohibitive surgical risk will be enrolled in a registry and undergo clinical and imaging surveillance.

\section{Study setting}

The study will be conducted at $>20$ academic hospitals with cardiac surgery centres across North America and Europe.

\section{Eligibility criteria}

Inclusion criteria

Age between 18 and 80 years, ascending aorta or aortic root aneurysm between $5.0 \mathrm{~cm}$ and $5.4 \mathrm{~cm}$ in maximal diameter on a contrast-enhanced CT scan. Individual surgeons have preferences as to whether large versus small, bicuspid versus trileaflet, men versus women, young versus elderly should have different thresholds for intervention. However, there are insufficient data to justify these clinical impressions, as reflected in current guidelines, which do not recommend different thresholds for these subgroups. Thus, all patients with nonheritable ATAA will be included in the study.

\section{Exclusion criteria}

- Patients who refused to be randomised, unable to give informed consent or unable to attend regular follow-up or be report with protocol.

- Patients with symptom(s) attributable to ascending aortic aneurysms.

- Previous cardiac surgery.

- Patients with nonaortic indication for cardiac surgery (eg, severe valvular insufficiency or stenosis).

- An AA expansion rate exceeding $0.5 \mathrm{~cm} /$ year.

- Involvement of arch aneurysms (no aneurysmal segments before the innominate artery).

- Ascending aortic and arch aneurysm with descending thoracic aorta involvement.

- Patients with known connective tissue disease (eg, Marfan syndrome, Loey-Dietz, Turner syndrome etc).

- Patients with possible genetic aortopathies (family history of aortic aneurysms/premature aortic dissections/ruptures).

- Patients with inflammatory arteritis (Takayasu's arteritis, syphilitic arteritis etc).

- Those who are deemed unfit for surgery (will not be included in the randomised control trial but will be included in the registry).

- severe pulmonary disease.

- $\mathrm{Cr} \geq 250$. 
Patients aged 18-79 years old with an ascending aortic aneurysm $\geq 5.0 \mathrm{~cm}$ on referral

Initial CT at the Aortic Clinic

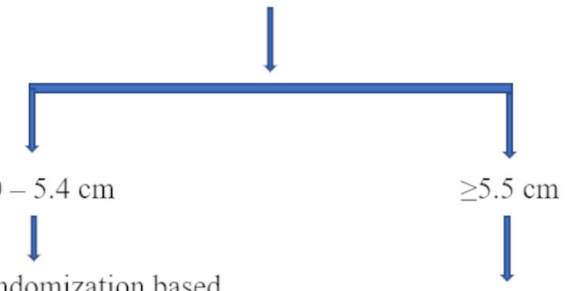

Eligible for randomization based on inclusion \& exclusion criteria?

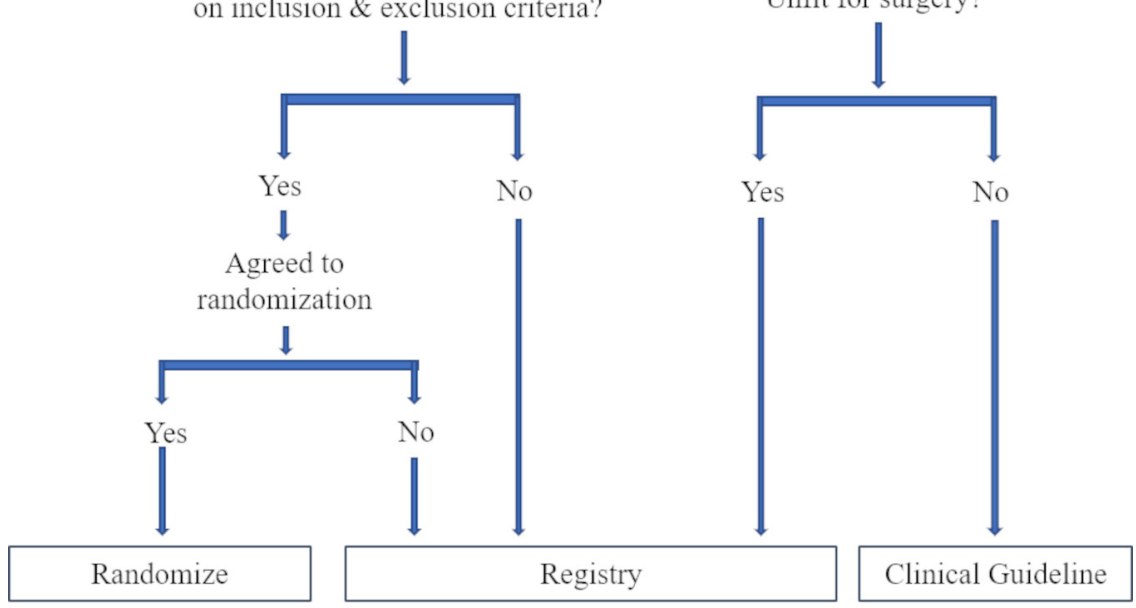

Figure 1 Treatment in Thoracic Aortic Aneurysm: Surgery versus Surveillance recruitment flowsheet.

- Child Pugh Class B or C.

- New York Heart Association (NYHA) III or IV.

- Myocardial infarction (MI) within the last 6 months

- Major surgical procedure or angioplasty within 3 months.

- Expected survival less than 5 years because of other disease (eg, invasive cancer).

Excluded patients will be invited to participate in the registry arm and will undergo protocol-driven clinical and imaging follow-up as per the surveillance arm of the study.

\section{Intervention}

In the surgery group, patients will undergo replacement of the aneurysmal aortic segment between 5.0 and $5.4 \mathrm{~cm}$ with a Dacron graft, within 12 weeks of randomisation. Patients may concomitantly undergo treatment of associated valvular and coronary artery disease, if present. The specific conduct of the aortic replacement and the need for concomitant cardiac procedures will be left to the discretion of the surgical team. Postoperative care will be provided according to protocols of the institution.

In the surveillance group, patients will undergo protocol-driven imaging studies. If patients have progression of aortic or other cardiac disease, they will undergo treatment as per established guidelines. The patients will undergo a CT scan of the chest at the first clinic visit then annually for the duration of the study. All CTs will be uploaded to a core CT imaging lab where the image will be reviewed by dedicated cardiovascular radiologists. If the aneurysm grows beyond $5.5 \mathrm{~cm}$ or the patient develop symptoms, the surgeon will consider the patient for surgery.

In both groups, patients will receive optimal medical therapy for their aneurysm according to established guidelines. $^{4-6}$

\section{Outcomes}

The primary outcome is all-cause mortality over the study period, with a minimum follow-up of 2 years.

The primary safety outcome will be incidence of perioperative stroke. Other secondary outcomes for this trial include operative and 30-day mortality following aortic surgery, incidence of acute aortic events, incidence of elective ascending aortic surgery in the surveillance group, aortarelated death, nonaorta related death, incidence of CVA during follow-up, annual ascending aortic growth rate (mm/year) in the surveillance group, quality of life at 1 year and 3 years assessed using the SF-36 questionnaire.

Outcome definitions are outlined in online supplemental appendix A.

\section{Sample size}

The study hypothesis is that a strategy of early surgery will lead to lower all-cause mortality at follow-up compared with surveillance. A framework, proposed by Barthel $e t a l,{ }^{19}$ for sample size calculation incorporating nonproportional hazards $(\mathrm{PH})$ and treatment changes 
including cross-over between treatment arms was used for determining the sample size. The framework is based on using piecewise exponential distributions to model the survival distributions. The approach has been implemented in the programme Analysis of Resources for Trials. For tractability, five periods will be taken of equal length (1year) with the number of patients at risk and occurrence of events in the two groups separately for each period. Modelling survival distributions over each of the periods allow us to take nonPH into account and cross-over that may vary over the time periods.

The event rate in the surveillance group is based on findings from the meta-analysis on the natural history of ascending aortic aneurysms. We found that the all-cause mortality was $1.99 \%$ /year in a cohort with small aneurysms (mean aortic diameter of $4.3 \mathrm{~cm}$ ). In contrast, the Yale University group, the largest single-centre cohort, reported a $5.5 \%$ /year incidence of mortality in patients with ascending aortic aneurysm between 5.0 and $5.9 \mathrm{~cm}$. We anticipate that the annual incidence of the primary outcome in the surveillance group will be between $1.99 \%$ and $5.5 \%$ and, therefore, an estimate of $3 \%$ will be used for the sample size calculation. For the surveillance group, survival probabilities per period are (1-0.03) k (ie, $0.9700,0.9409,0.9127,0.8853,0.8587)$ and incorporating $10 \%$ cross-over probabilities are $0.9718,0.9465,0.9227$, $0.9002,0.8786$.

In the surgery group, we estimate that the 30-day mortality can vary between $1 \%$ and $2 \%$. Data from large volume aortic centres in the literature as well as from the nominated principal investigator (PI)'s institution support a 30-day mortality of $1 \%$. We have assumed a conservative 30-day mortality estimate of $2 \%$. Following ascending aortic replacement, we anticipate a $1 \% /$ year incidence of nonaortic mortality in the surgical group. This has been corroborated in a review of long-term outcomes following aortic surgery at the Ottawa Heart Institute where survival in study eligible patients $(n=506)$ following aortic surgery was $93 \% \pm 2 \%$ at 10 years. For the surgery group, survival probabilities per period are (1-0.01) k (ie, 0.9900, 0.9801, $0.9703,0.9606,0.9510$ ).

A two-sided log-rank test with a sample size of 278 subjects per group achieves an $80 \%$ power at a 0.047 significance level (accounting for one interim analysis) to detect a difference in the proportion surviving in the early selective surgery group of 0.99 compared with the proportion surviving in the surveillance group of 0.97 . This assumes uniform accrual over a

3-year period followed by minimum of 2 years of follow-up. An anticipated loss to follow-up rate is $10 \%$, and to account for this loss, the total sample size will be 618 subjects (556/0.9), 309 in the surgery arm and 309 in the surveillance arm. With the sample size of 618 , and with the expected no loss in the perioperative period, for the primary safety outcome of perioperative stroke, the power is $80 \%$ to detect a difference in perioperative stroke of $2.5 \%$, a difference that is considered clinically important to detect.

\section{Recruitment}

Recruitment will be conducted among all patients referred for ascending aortic surgery at participating centres who meet the inclusion criteria. Participating centres have a dedicated thoracic aortic clinic in which patients are followed by dedicated aortic surgeons.

\section{Allocation}

Research coordinators will screen patients seen at the thoracic aortic clinic for enrolment eligibility. If the patient is eligible based on inclusion and exclusion criteria, the research coordinator will consent the patient to participate in the study (online supplemental appendix B). After consent is obtained, the research coordinator will obtain randomisation through a centralised web-based randomisation tool en-site, which will assign the patient to either surgery or surveillance in a 1:1 ratio. Allocation sequence is generated completely at random and is not dependent on the previous treatment assignment. The patient, research coordinator and the surgeon will not be blinded to the intervention assignment.

Aortic size will be determined using a double-oblique measurement of maximal ascending aortic diameter orthogonal to the direction of blood flow using contrastenhanced CT scan based on American Society of Radiology Guidelines. ${ }^{4}$ Patients presenting with echocardiograms or MRI will undergo a CT scan prior to determining eligibility. Informed consent will be obtained, and a pre-randomisation checklist will be completed. Patients will then be randomised using a web-based randomisation tool. Randomisation will be stratified for the anticipated need for aortic root intervention as this is an established risk factor for increased operative risk. ${ }^{20}$ Additional stratification variables will include bicuspid aortic valve and study site.

\section{Follow-up}

Participant timeline is outlined in table 1. Patients in the surgery group will undergo routine postoperative follow-up between 4 and 8 weeks after surgery and then annually from randomisation until study close. For patients unable to attend in person, a research coordinator will conduct a telephone follow-up. A contrastenhanced, gated thoracic CT scan will be obtained at 1 year postsurgery. If this CT scan identifies no anastomotic site abnormalities and no aortic segments greater than $4.5 \mathrm{~cm}$, then no further CT scans will be obtained in this group to minimise radiation exposure. Patients will be followed for a minimum of 2 years or until the study ends.

Patients randomised to the surveillance group will undergo a clinical assessment to review and optimise medical therapy and a contrast-enhanced, ECG-gated thoracic CT scan annually from the date of randomisation ( \pm 4 weeks) for the duration of the study. All CT scans will be uploaded to a CT imaging core lab (University of Calgary) where images will be reviewed and reported by a study radiologist using TeraRecon (Aquarius iNtuition, 
Table 1 Participant timeline of TITAN SvS trial

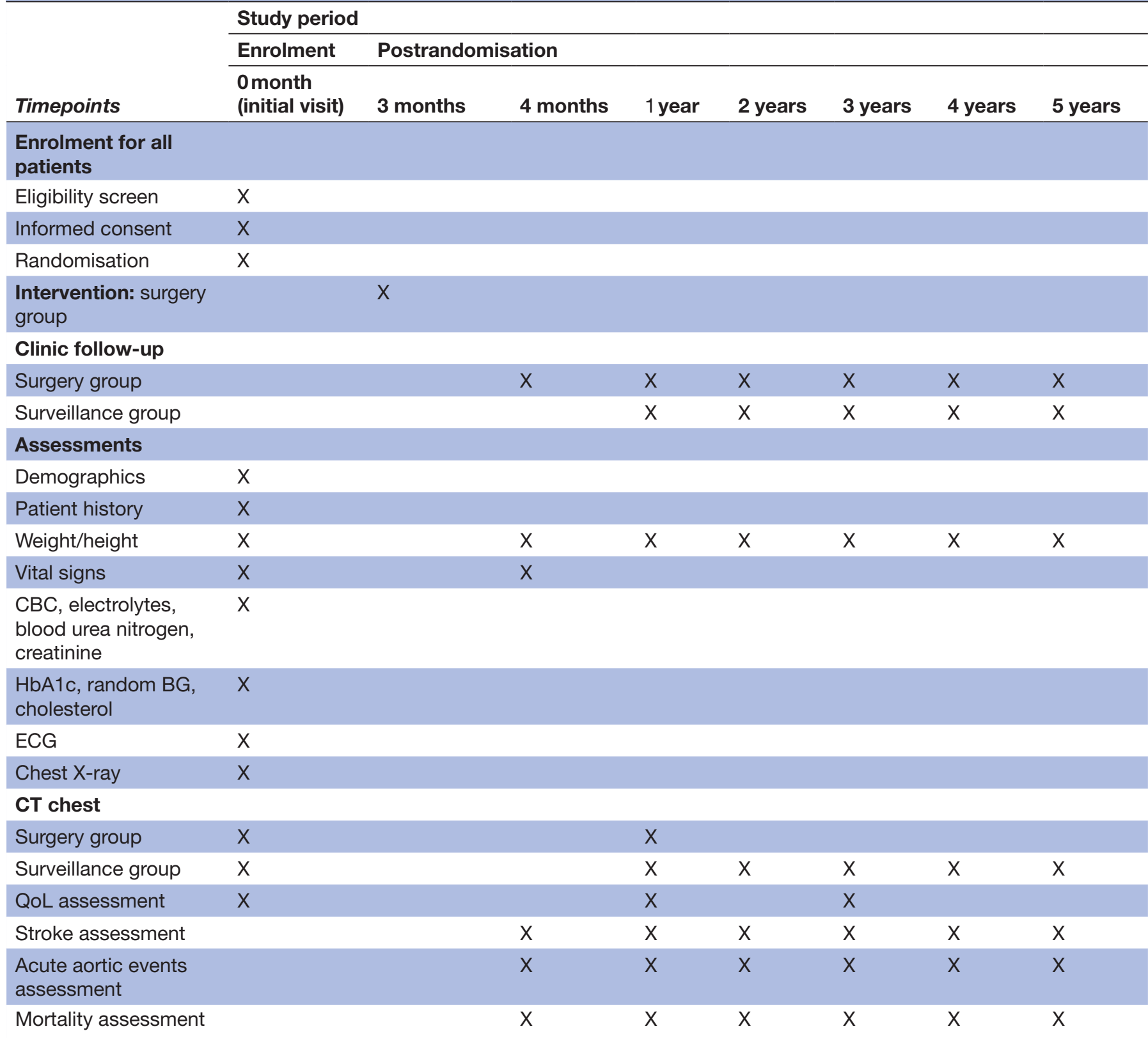

BG, blood glucose; BUN, blood urea nitrogen; CBC, complete blood count; Cr, creatinine; QoL, quality of life.

California). In patients with pre-existing chronic kidney disease that did not meet exclusion criteria, non-contrast CT will suffice given the consistent expertise at the core lab. Local CT readings will be used to determine inclusion into study. All subsequent aortic readings will be done by the imaging core lab. To ensure concordance and to examine variance between study site and core lab aortic measurements, baseline CT scans will also be reviewed by the core lab and measurements compared with study site measurements. In case of aortic or cardiac disease progression, the patient will undergo guideline-directed surgical therapy. Similarly, patients will be followed for a minimum of 2 years or until the study ends.

\section{Registry arm}

Patients will be enrolled into the Non-operative Registry (Sub-Study arm) rather than the RCT in three circumstances: (1) if the patient has an ascending aortic aneurysm between $5.0 \mathrm{~cm}$ to $5.4 \mathrm{~cm}$ but does not meet eligibility criteria for enrolment into the trial, (2) if the patient meets eligibility criteria for the trial but refuses to participate in randomisation and (3) if the patient has an ascending aortic aneurysm $\geq 5.5 \mathrm{~cm}$ and is deemed a nonoperable candidate. Baseline information as indicated above, including a full history, vitals, complete physical examination, and an initial CT scan will also be obtained in the first clinic visit as per the standard of 
care. Patients will be clinically followed each year with a CT scan to monitor the status of their ascending aortic aneurysm, until the end of the study, the occurrence of an aortic event (aortic dissection or aortic rupture) or death.

\section{Data collection methods}

Patient data will be collected electronically by web-based case report form through DACIMA database. All study site personnel will be trained on the protocol, and all studyrelated tasks and procedures that they have been delegated by the local PI. This training will be documented in a protocol training $\log$.

To promote completeness of follow-up, telephone follow-up will be conducted by a research coordinator if the patient is unable to attend annual clinical follow-up in person. Quality of life assessment by SF-36 can also be completed by telephone at 1-year and 3-year follow-up. If there is a deviation from the protocol, the date, description and reason for protocol deviation will be reported. In addition, actions taken to prevent future protocol deviation, if any, will be documented. All major protocol deviations will be reported to the local Research Ethics Board (REB).

\section{Statistical methods}

The analysis will follow the intention-to-treat principle.

\section{Primary analysis}

The patient groups will be compared on the time-to-the primary outcome, all-cause mortality, using the log-rank test and described using Kaplan-Meier curves. If there are baseline differences, the Cox PH model will be used to compare groups adjusting for baseline variables. The interaction of treatment by stratification variables will be evaluated within the model. The assumption of $\mathrm{PH}$ will be assessed using the log-negative-log plot of the Kaplan-Meier estimator, a plot of Schoenfeld residuals as a function of time and consideration of the interactions of selected covariates with time. If the $\mathrm{PH}$ assumption is violated, then modification of the model for non-PH will be considered including adding interaction of covariate(s) with function of time and stratification models. For interactions with function of time, an interaction is added of a covariate for which the $\mathrm{PH}$ assumption is violated. If the interaction is statistically significant, the effect of the given covariate is not constant over time and including the interaction in the model enables interpretation of the parameter estimate taking this into account. For the stratified model, subgroups based on a categorical variable chosen in that it interacts with time and the $\mathrm{PH}$ assumption is violated for this variable will be considered.

\section{Secondary analyses}

For the primary safety outcome perioperative stroke, the patient groups will be compared using the $\chi^{2}$ test. If there are baseline differences, then a logistic regression model will be used to compare patient groups adjusting for baseline variables. For the continuous outcome of quality of life, a 2-way analysis of variance will be considered with the between factor time (elective surgery group vs surveillance group) and the within factor time (1 vs 3 years). If there are any clinically important imbalances in baseline variables, then an analysis of covariance will be used to compare patient groups over time on quality of life adjusting for these baseline variables. For the other secondary dichotomous outcomes, proportions will be calculated and when comparisons are made between the patient groups, they will be compared using the $c \chi^{2}$ test. If there are baseline differences, then a logistic regression model will be used to compare the patient groups adjusting for these baseline variables. In the surveillance group, the point estimates and $95 \%$ CIs will be calculated for the incidence of elective ascending aortic surgery and the annual ascending aortic growth rate.

\section{Subgroup analyses}

Three prespecified subgroup analyses will be performed that address important areas of controversy in the management of aortic disease. We will examine the relationship between indexed aortic size (aortic diameter/body surface area- $\mathrm{cm} / \mathrm{m}^{2}$ ) and primary outcome to determine whether indexed aortic size is a better predictor than absolute size for the primary endpoint. We will examine the relationship between the primary outcome and aortic valve phenotype (bicuspid vs tricuspid) as there is suggestion in the literature that size thresholds for surgery should be lower for patients with bicuspid aortic valve disease. ${ }^{4}{ }^{12}$ Finally, the PI and coapplicants have recently shown women with ascending aortic aneurysms have a faster growth rate, which may lead to increasing aortic complications. ${ }^{21}$ women have also been shown to have higher mortality rates related to aortic disease. ${ }^{22}$ We will examine the relationship between sex and study intervention by evaluating an interaction term (sex $\times$ intervention) to determine whether the effect of intervention is different in men vs women.

\section{Monitoring}

A data safety monitoring board (DSMB) is independent of the investigators and whose members collectively have adequate expertise in aortic aneurysms, cardiac surgery and biostatistics, they will convene regularly to review trial safety events and outcomes data. The DSMB will keep minutes of their meetings and report to the investigators whether any modifications to the trial are recommended.

An interim analysis of the primary and secondary endpoints will be conducted by an unblinded study statistician when $50 \%$ of subjects have been recruited using the O'Brien Fleming procedure for multiple testing at a significance level of $\mathrm{p}=0.003$.

Adverse events (AE) will be recorded for study subjects while they are enrolled in the trial. Serious AE that occur at any time after the inclusion of the subject in the study (defined as the time when the subject is randomised) up to 30 days after the subject completes or discontinues their participation in the trial, whichever is first, must be reported. 
The incidence of major adverse cardiovascular events (mortality, myocardial infarction, CVA and acute aortic events) will be recorded (and noted as AE) for trial subjects in the 5-year period ( \pm 30 days) after randomisation.

The following variables will be collected for each AE:

- AE description.

- The date when the AE started and stopped.

- Whether the AE is serious or not, and rationale (ie, hospitalisation, probably cause of death).

- Investigator causality rating against the study intervention.

- Investigator assessment of whether event was expected or not.

- Whether AE caused subject's withdrawal from study.

- Patient outcome.

Events that are serious, unexpected and possibly or probably related to the study intervention will be reported to the DSMB.

\section{Access to data}

Individual REB will have access to anonymised data at their discretion, as per REB policy. However, no thirdparty investigators or corporate bodies will have access to study data prior to synthesis and dissemination.

\section{Patient and public involvement}

There were no funds or time allocated for patient and public involvement, however, we will invite patients to help us develop our dissemination strategy.

\section{ETHICS AND DISSEMINATION}

\section{Research ethics approval and protocol amendments}

The REB has granted approval to the study at 14 participating institutions; inclusion of further enrolling centres in the future may be considered (online supplemental appendix C). Any protocol amendments will be communicated to the REB at the respective institution.

\section{Consent or assent}

The research coordinator will meet with eligible patients in person to explain the trial and obtain consent for participation. Additional consent will need to be obtained for collection of aortic tissue sample from the patient during surgery.

\section{Confidentiality}

Each participating patient will be assigned a study ID for which the data are collected under. The Master List that match the patient's name to their research ID will be stored in a password-protected research drive accessible only to personnel involved in the conduct of the trial. All data are collected through the centralised web-based case report form and will be accessible only to those participating in the collection and analysis of the data.

\section{Declaration of interests}

There is no conflict of interest for Dr Guo, Dr Appoo, Dr Wells, Ms Fortier and Dr Boodhwani. Dr Ououznian is on the North American Advisory Board (consultant position) for Medtronic; Dr Michael Chu receives Speaker's honoraria from Medtronic, Edwards Lifesciences, Boston Scientific, Terumo Aortic and Abbott Vascular.

\section{Ancillary and Post-trial care}

Patients with ascending aortic aneurysm between 5.0 and $5.4 \mathrm{~cm}$ will be randomised to surgery or surveillance. Therefore, patients randomised to the surgical arm will be subjected to the risks and discomforts of elective ascending aortic surgery. In experienced centres, the operative mortality and risk of postoperative stroke rate at an experienced centre for composite aortic root replacement is $1.9 \%$ and $1.4 \%$, respectively, and $0 \%$ and $1 \%$ for root sparing ascending aortic replacement. ${ }^{20}{ }^{23}$ At the nominated PI centre, 303 consecutive patients undergoing elective ascending aortic surgery (with or without aortic root replacement), 30-day mortality and stroke occurred at rate of $1.2 \%$ and $1 \%$, respectively (unpublished data). Notably, in our survey of current clinical practice, about half of the respondents offer surgery at $5.0 \mathrm{~cm}$ and half at $5.5 \mathrm{~cm}$ for the same patient characteristics. Thus, due to clinical equipoise and practice variation, the number of trial eligible patients in the community receiving SvS would approximately be equal, thereby not increasing overall risk to patients. Therefore, post-trial care will continue to be consistent with that recommended by current guideline, and the patients will be followed accordingly.

\section{Dissemination policy}

The trial is registered with www.clinicaltrials.org, and the protocol of the trial will be published. The result of the trial will be presented at national and international conferences and published in relevant journals, regardless of the finding of the trial. Authors of the publications will include those who participated in the design and maintenance of the trial, investigators from participating centres who enrolled patients into the trial, those who provided critical guidance for the trial and those who participated in the analysis of results and drafting of the manuscript.

\section{Author affiliations \\ ${ }^{1}$ Cardiac Surgery, University of Ottawa Heart Institute, Ottawa, Ontario, Canada \\ ${ }^{2}$ Cardiac Surgery, University of Calgary, Calgary, Alberta, Canada \\ ${ }^{3}$ School of Epidemiology and Public Health, University of Ottawa, Ottawa, Ontario, Canada \\ ${ }^{4}$ Cardiovascular Research Methods Centre, University of Ottawa Heart Institute, Ottawa, Ontario, Canada \\ ${ }^{5}$ Cardiac Surgery, Western University Schulich School of Medicine and Dentistry, London, Ontario, Canada \\ ${ }^{6}$ Cardiac Surgery, University Health Network, Toronto, Ontario, Canada}

Contributors All authors contributed to: the design of the trial; drafted or provided revision of critical components of the manuscript; gave final approval of the protocol; agreed to be accountable for all aspects of the work in ensuring that questions related to the accuracy or integrity of any part of the work are appropriately investigated and resolved. Specific author contribution: MHG: protocol development; JJA: co-principal investigator; GAW: statistician; MC: advisory to trial design and manuscript revision; M0: advisory to trial design and manuscript 
revision; JF: advisory to trial design and ethics approval; MB: co-principal investigator.

Funding The TITAN: SvS trial is funded by CIHR Project Grant.

Competing interests Dr Ououznian is on the North American Advisory Board (consultant position) for Medtronic Inc; Dr Michael Chu receives Speaker's honoraria from Medtronic, Edwards Lifesciences, Boston Scientific, Terumo Aortic, and Abbott Vascular.

Patient consent for publication Not required.

Provenance and peer review Not commissioned; peer reviewed for ethical and funding approval prior to submission.

Supplemental material This content has been supplied by the author(s). It has not been vetted by BMJ Publishing Group Limited (BMJ) and may not have been peer-reviewed. Any opinions or recommendations discussed are solely those of the author(s) and are not endorsed by BMJ. BMJ disclaims all liability and responsibility arising from any reliance placed on the content. Where the content includes any translated material, BMJ does not warrant the accuracy and reliability of the translations (including but not limited to local regulations, clinical guidelines, terminology, drug names and drug dosages), and is not responsible for any error and/or omissions arising from translation and adaptation or otherwise.

Open access This is an open access article distributed in accordance with the Creative Commons Attribution Non Commercial (CC BY-NC 4.0) license, which permits others to distribute, remix, adapt, build upon this work non-commercially, and license their derivative works on different terms, provided the original work is properly cited, appropriate credit is given, any changes made indicated, and the use is non-commercial. See: http://creativecommons.org/licenses/by-nc/4.0/.

\section{ORCID iD}

Munir Boodhwani http://orcid.org/0000-0003-2815-8697

\section{REFERENCES}

1 Hagan PG, Nienaber CA, Isselbacher EM, et al. The International registry of acute aortic dissection (IRAD): new insights into an old disease. JAMA 2000;283:897-903.

2 Pape LA, Awais M, Woznicki EM, et al. Presentation, Diagnosis, and Outcomes of Acute Aortic Dissection: 17-Year Trends From the International Registry of Acute Aortic Dissection. J Am Coll Cardiol 2015;66:350-8.

3 Davies RR, Goldstein LJ, Coady MA, et al. Yearly rupture or dissection rates for thoracic aortic aneurysms: simple prediction based on size. Ann Thorac Surg 2002;73:17-28.

4 Hiratzka LF, Bakris GL, Beckman JA, et al. 2010 ACCF/AHA/AATS/ ACR/ASA/SCA/SCAI/SIR/STS/SVM guidelines for the diagnosis and management of patients with thoracic aortic disease: a report of the American College of cardiology Foundation/American heart association Task force on practice guidelines, American association for thoracic surgery, American College of radiology, American stroke association, society of cardiovascular Anesthesiologists, Society for cardiovascular angiography and interventions, society of interventional radiology, society of thoracic surgeons, and Society for vascular medicine. Circulation 2010;121:e266-369.

5 Erbel R, Aboyans V, Boileau C, et al. 2014 ESC guidelines on the diagnosis and treatment of aortic diseases: document covering acute and chronic aortic diseases of the thoracic and abdominal aorta of the adult. The task force for the diagnosis and treatment of aortic diseases of the European Society of cardiology (ESC). Eur Heart $J$ 2014;35:2873-926.

6 Boodhwani M, Andelfinger G, Leipsic J, et al. Canadian cardiovascular Society position statement on the management of thoracic aortic disease. Can J Cardiol 2014;30:577-89.

7 Appoo JJ, Bozinovski J, Chu MWA, et al. Canadian cardiovascular Society/Canadian Society of cardiac Surgeons/Canadian Society for vascular surgery joint position statement on open and endovascular surgery for thoracic aortic disease. Can J Cardiol 2016;32:703-13.

8 Guo MH, Appoo JJ, Saczkowski R, et al. Association of mortality and acute aortic events with ascending aortic aneurysm: a systematic review and meta-analysis. JAMA Netw Open 2018;1:e181281.

9 Mori M, Shioda K, Wang X, Fort Lauderdale. Comtemporary characteristics and risk profiles for proximal thoracic aortic surgeries for aortic Aneurym: analysis of the STS national ACSD. Society of Thoracic Surgeons Annual Meeting 2018, Florida, USA, 2018.

10 Pape LA, Tsai TT, Isselbacher EM, et al. Aortic diameter $>$ or $=5.5$ $\mathrm{cm}$ is not a good predictor of type A aortic dissection: observations from the International Registry of Acute Aortic Dissection (IRAD). Circulation 2007;116:1120-7.

11 Mansour AM, Peterss S, Zafar MA, et al. Prevention of aortic dissection suggests a diameter shift to a lower aortic size threshold for intervention. Cardiology 2018;139:139-46.

12 Rylski B, Blanke P, Beyersdorf F, et al. How does the ascending aorta geometry change when it dissects? J Am Coll Cardiol 2014;63:1311-9.

13 Chaikof EL, Brewster DC, Dalman RL, et al. SVS practice guidelines for the care of patients with an abdominal aortic aneurysm: Executive summary. J Vasc Surg 2009;50:880-96.

14 Ashton HA, Buxton MJ, Day NE, et al. The multicentre aneurysm screening study (mass) into the effect of abdominal aortic aneurysm screening on mortality in men: a randomised controlled trial. Lancet 2002;360:1531-9.

15 Lederle FA, Wilson SE, Johnson GR, et al. Immediate repair compared with surveillance of small abdominal aortic aneurysms. $N$ Engl J Med 2002;346:1437-44.

16 Norman PE, Jamrozik K, Lawrence-Brown MM, et al. Population based randomised controlled trial on impact of screening on mortality from abdominal aortic aneurysm. BMJ 2004;329:1259.

17 , Powell JT, Brady AR, et al, United Kingdom Small Aneurysm Trial Participants. Long-Term outcomes of immediate repair compared with surveillance of small abdominal aortic aneurysms. N Engl J Med 2002;346:1445-52.

18 Scott RAP, Bridgewater SG, Ashton HA. Randomized clinical trial of screening for abdominal aortic aneurysm in women. $\mathrm{Br} J$ Surg 2002;89:283-5.

19 Barthel FM-S, Babiker A, Royston P, et al. Evaluation of sample size and power for multi-arm survival trials allowing for non-uniform accrual, non-proportional hazards, loss to follow-up and cross-over. Stat Med 2006;25:2521-42.

20 Mok SCM, Ma W-G, Mansour A, et al. Twenty-Five year outcomes following composite graft aortic root replacement. J Card Surg 2017;32:99-109.

21 Cheung K, Boodhwani M, Chan K-L, et al. Thoracic aortic aneurysm growth: role of sex and aneurysm etiology. J Am Heart Assoc 2017;6:e003792.

22 Chung J, Stevens L-M, Ouzounian M, et al. Sex-Related differences in patients undergoing thoracic aortic surgery. Circulation 2019;139:1177-84.

23 Peterss S, Charilaou P, Dumfarth J, et al. Aortic valve disease with ascending aortic aneurysm: impact of concomitant root-sparing (supracoronary) aortic replacement in nonsyndromic patients. $J$ Thorac Cardiovasc Surg 2016;152:791-8. 